\title{
A Nonsense Mutation in the SCN9A Gene in Congenital Insensitivity to Pain
}

\author{
Mazen Kurban ${ }^{\mathrm{a}}$ Muhammad Wajid $^{\mathrm{a}}$ Yutaka Shimomura $^{\mathrm{a}}$ \\ Angela M. Christiano a, b \\ Departments of ${ }^{\mathrm{a}}$ Dermatology and ${ }^{\mathrm{b}}$ Genetics and Development, Columbia University, New York, N.Y., USA
}

\section{Key Words}

Erythromelalgia $\cdot$ SCN9A gene $\cdot$ Anosmia $\cdot$ Na1.7 channels

\begin{abstract}
Background: Congenital insensitivity to pain (CIP) (OMIM 243000) is a rare autosomal-recessive disorder. Clinically, CIP is characterized by insensitivity to all modalities of pain except neuropathic pain, and recurrent injuries frequently go unnoticed. CIP is caused by mutations in the SCN9A gene encoding for the Na1.7 channel. Methods: We analyzed the DNA from members of a consanguineous Pakistani family for mutations in the SCN9A gene through direct sequencing after performing linkage studies. Results: We identified a novel missense mutation designated R523X in all affected individuals. A screening assay ruled out the possibility of polymorphism. Conclusion: We identified a novel mutation in the Na1.7 channel leading to CIP, extending the spectrum of mutations in the Na1.7 channel, and enhancing our understanding of the physiology of pain.
\end{abstract}

Copyright $\odot 2010$ S. Karger AG, Basel

\section{KARGER}

Fax +41613061234 E-Mail karger@karger.ch www.karger.com
() 2010 S. Karger AG, Basel

\section{Introduction}

Pain is a highly conserved sensory modality among all vertebrate species which confers protection against noxious and dangerous stimuli. Loss of pain sensation is associated with multiple injuries that compromise survival. On the other hand, hypersensitivity to pain lies on the other extreme of the spectrum and would also adversely affect individuals, as they would perceive several nonharmful stimuli as being noxious. Pain is categorized into several subtypes, including mechanical, visceral, inflammatory, thermal, chemical and neuropathic.

Voltage-gated sodium channels are the channels responsible for the conversion of mechanical or chemical stimuli into electrical signals within excited cells [1]. These channels are composed of functional pore-forming $\alpha$ subunits and auxiliary $\beta$ subunits [2]. To date, nine different sodium channels encoded by nine different genes designated $S C N(1-9 A)$ have been identified. Inactivation of SCN1A and SCN2A [3] genes have been associated with epilepsy, $S C N 4 A$ is associated with myotonia disorders [4], SCN5A is associated with Brugada syndrome (OMIM 601144) [5], which is characterized by cardiac arrhythmias in young people, and SCN8A is associated with cerebellar atrophy and ataxia [6]. SCN9A is

Angela M. Christiano, $\mathrm{PhD}$

Department of Dermatology, Columbia University

Russ Berrie Medical Center, 1150 St. Nicholas Avenue

New York, NY 10032 (USA)

Tel. +1 212851 4850,Fax +1 212851 4810,E-Mail amc65@ columbia.edu 
associated with indifference to pain in cases with inactivating mutations [7], and the opposite phenotype, hyperalgesia, in cases of activating mutations $[8,9]$.

Nav1.7, a tetrodotoxin-sensitive sodium channel encoded by the SCN9A gene, and Nav1.8, a tetrodotoxininsensitive sodium channel, are both expressed in the peripheral nervous system and mainly in the dorsal root ganglia and are closely interconnected. They are implicated in pain perception including inflammatory and mechanical pain [10], but not neuropathic pain [11]. Despite the discovery of these two channels and their modes of action, the pathophysiology of pain perception is incompletely understood. To date, mutations in Nav1.7 (OMIM 603415) have been implicated in three pain-related syndromes in humans.

Primary erythromelalgia (PE) (OMIM 133020) and paroxysmal extreme pain disorder (PEPD) (OMIM 167400) are two autosomal-dominant disorders characterized by severe pain. PE involves primarily the hands and feet and to a lesser extent the ears and nose. It usually presents in the first decade of life with severe episodic erythema, pain and flushing that respond well to cooling; symptoms worsen with age [12, 13]. PEPD is characterized by chronic episodic pain attacks starting soon after birth and involving the rectum with radiation to the lower extremities bilaterally triggered mainly by defecation, accompanied by ocular and mandibular pain. Unlike attacks of PE, these attacks are responsive to carbamezepine [9]. Symptoms usually improve with age. Pain in the two syndromes is of the inflammatory type, and is caused by activating mutations in the SCN9A gene $[14,15]$.

In contrast to PE and PEPD, congenital insensitivity to pain (CIP) (OMIM 243000) is an autosomal-recessive disease caused by inactivating mutations in the Nav1.7 channel [7]. Patients present during the first decade of life with lack of pain perception, but with preservation of all other sensory modalities including normal touch perception, proprioception, itch, warm and cold temperature discrimination and vibration. Individuals with CIP generally present with injuries involving the lips and tongue due to asymptomatic injuries during the first few years of life $[7,16]$. Bone fractures are also asymptomatic and affected individuals are not aware of these fractures until a limp develops. An accompanying feature among several patients with CIP is anosmia or hyposmia $[16,17]$.

To date, only a limited number of inactivating mutations in the SCN9A gene have been found, among which the majority were nonsense mutations resulting in CIP. Here we present a family from Pakistan with CIP and a novel mutation in the SCN9A gene, further extending the spectrum of mutations in this gene and providing a framework for the better understanding of pain physiology.

\section{Materials and Methods}

DNA Extraction

After obtaining informed consent, we collected peripheral blood samples from family members and 100 population-matched unrelated healthy control individuals in EDTA-containing tubes under institutional approval and in adherence to the Declaration of Helsinki Principles. Genomic DNA was isolated from these samples according to standard techniques.

Haplotype Analysis

In order to determine if the phenotype of patients linked to the SCN9A gene, we performed haplotype analysis using two microsatellite markers, D2S1379 and D2S2330, closely linked to the gene. The amplification conditions for each PCR were $94^{\circ} \mathrm{C}$ for $2 \mathrm{~min}$, followed by 35 cycles of $94^{\circ} \mathrm{C}$ for $30 \mathrm{~s}, 55^{\circ} \mathrm{C}$ for $30 \mathrm{~s}$, and $72^{\circ} \mathrm{C}$ for $30 \mathrm{~s}$, with a final extension at $72^{\circ} \mathrm{C}$ for $7 \mathrm{~min}$. The amplified PCR products were run on an $8 \%$ polyacrylamide gel and genotyping was done by visual inspection.

Mutation Analysis

All exons of the SCN9A gene with adjacent sequences of exonintron borders were amplified by PCR with primers and conditions described previously [7]. The amplified PCR products were directly sequenced in an ABI Prism 310 Automated Sequencer, using the ABI Prism Big Dye Terminator Cycle Sequencing Ready Reaction Kit (PE Applied Biosystems).

\section{Screening Assays}

We performed a PCR and restriction endonuclease digestion assay for the novel mutation. We amplified exon 10 of the SCN9A gene using the same primers for mutation analysis. This was followed by $\mathrm{Cac} 8 \mathrm{I}$ digestion at $37^{\circ} \mathrm{C}$ for $2 \mathrm{~h}$. The product fragments were separated on $2 \%$ agarose gel.

\section{Results}

\section{Patients}

The pattern of inheritance of CIP in this family was autosomal-recessive (fig. 1a). By history, patients reported having experienced no pain from trauma including fractures, burns, and accidental tongue or lip biting, with some individuals losing the distal parts of the tongue (fig. 2a). In addition, none reported having abdominal or chest pain events. All patients reported the ability to discriminate among different odors. On physical examination, all patients had injuries over the lips and tongues. Neurological examination was consistent with defective perception of pain stimuli, while the other sensory mo- 

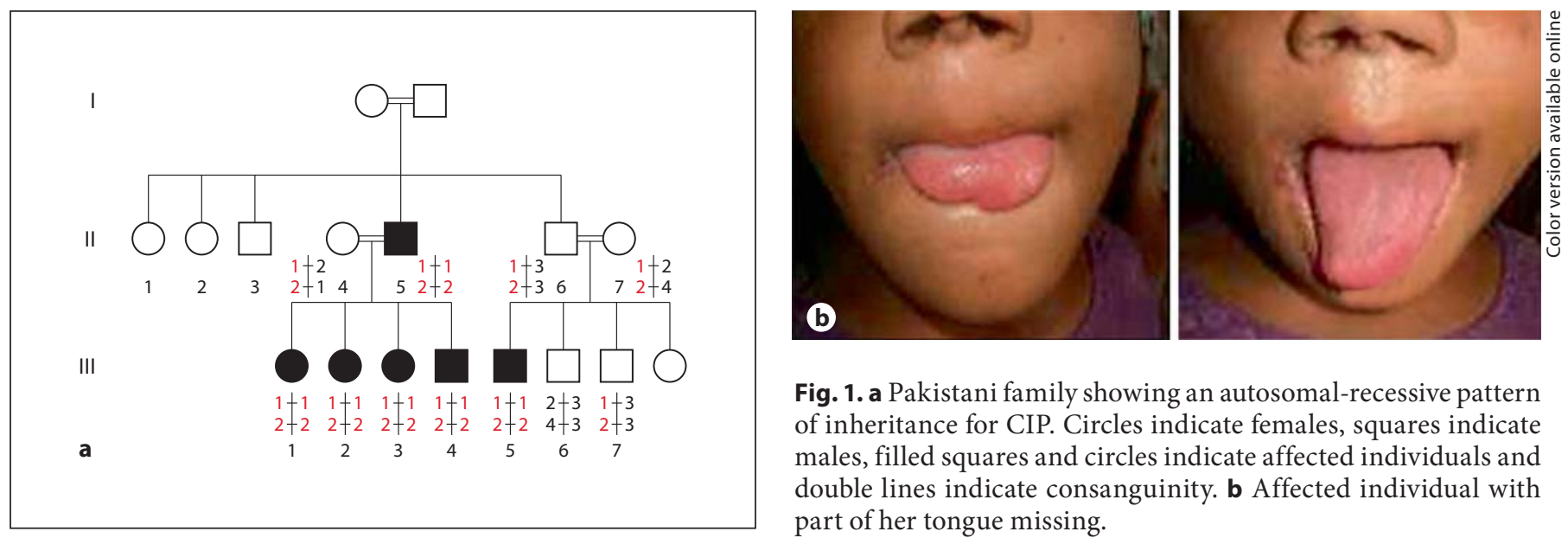

Fig. 1. a Pakistani family showing an autosomal-recessive pattern of inheritance for CIP. Circles indicate females, squares indicate males, filled squares and circles indicate affected individuals and double lines indicate consanguinity. b Affected individual with part of her tongue missing.

Fig. 2. a Affected individuals (II-5, III-1, III-2, III-3, III-4, and III-5) show a homozygous mutation at position $1567 \mathrm{C}>\mathrm{T}$ leading to substitution of arginine by a stop codon. Carrier individuals from the same family (II-4, II-6, II-7, and III-7) are heterozygous for the mutation. Control individuals are homozygous for the wildtype allele. $\mathbf{b}$ The restriction enzyme Cac8I was used for the screening assay for the mutation R523X. The enzyme is expected to digest the wild-type allele into two fragments, while the mutant allele is resistant to the digestion. The smaller fragment is not seen.

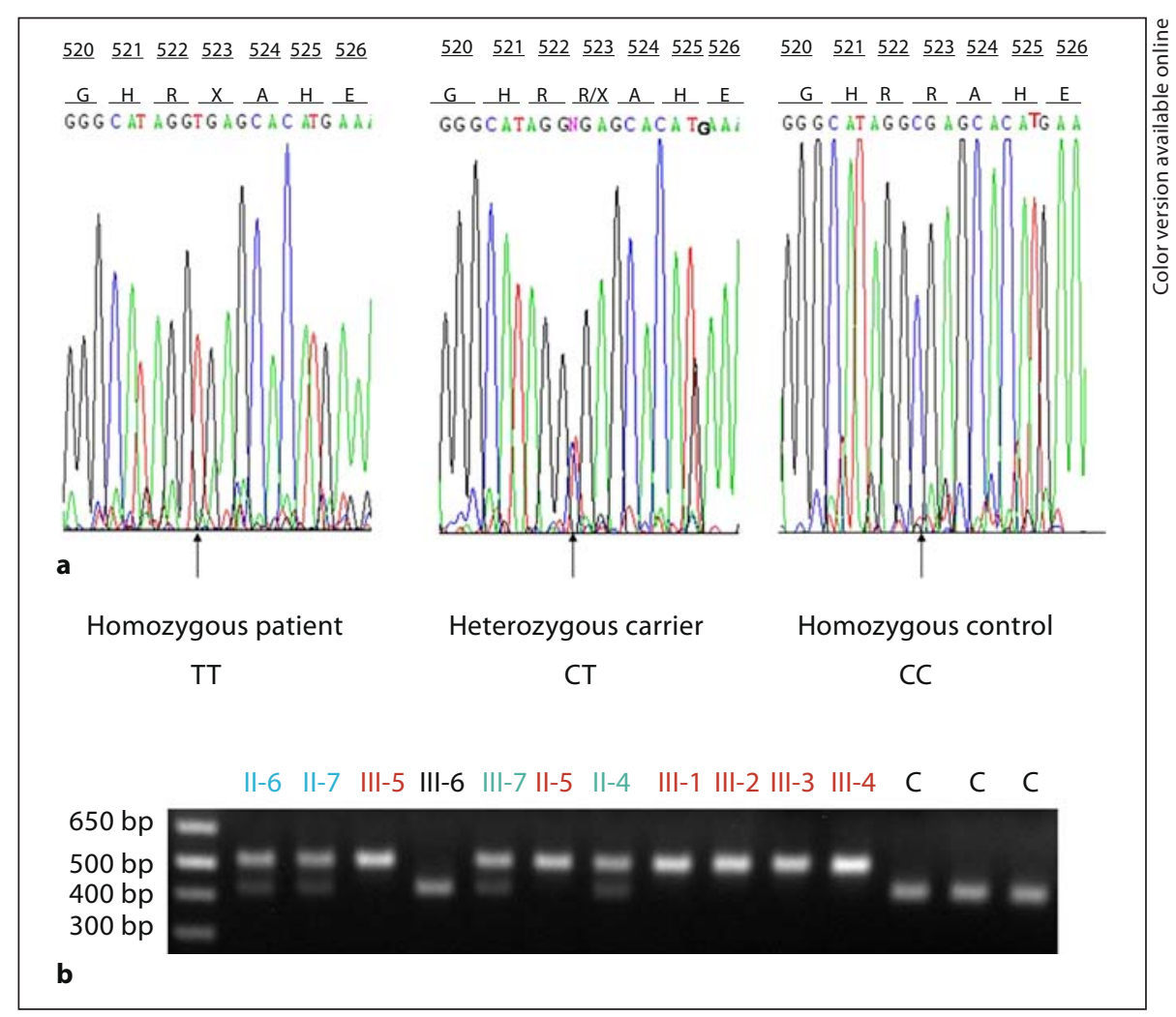

dalities including touch, tickle and pressure, warm and cold temperatures, and proprioception were within normal limits. Motor examination and cranial nerve examination including the olfactory nerve were normal.

\section{Genetic Analysis}

Haplotype analysis revealed phenotype linkage to the region on chromosome 2 spanning the SCN9A gene (fig. 1). A homozygous nonsense mutation in exon 10 of the SCN9A gene was identified at position 1567 designated R523X and was found in the homozygous state among all affected individuals (fig. 2a). Unaffected individuals were either heterozygous for the mutation or carried the wild-type allele. Screening assays performed in 100 control individuals (200 chromosomes) from the same population ruled out the possibility of polymorphism (fig. 2b). 


\section{Discussion}

The molecular pathways controlling pain perception are highly complex. Given this complex neural circuitry, it is surprising that an alteration in a single sodium channel (Na1.7) can have such profound effects leading to loss of all pain sensation (with the exception of neuropathic pain) when deactivated. This has great implications, mainly at the pharmacological level, as finding antagonists for Na1.7 would have great impact in the field of analgesia.

Na1.7 channels function mainly as gatekeeper channels [18], such that they amplify small signals and bring up neurons to certain voltages, which in turn activate the Na1.8 channels that have a much higher threshold for activation. Na1.8 channels then produce most of the current necessary for the generation of action potentials and therefore transduction of pain [19]. The pathway of pain mediated through both channels is most likely to be acting at the level of peripheral nociceptive transmission since these channels are mainly expressed in the peripheral nervous system, although a central nervous system contribution cannot be entirely ruled out, as there is some expression of $\mathrm{Na} 1.7$ and $\mathrm{Na} 1.8$ channels at these sites [20].

In cases of PE and PEPD, the mutations detected in the Na1.7 are missense mutations and result in lowering of the threshold of the channels to be activated by small depolarization events, thus enhancing the activity of the channels [21]. Moreover, these mutations slow the deactivation of the channels, keeping them open for an increased duration and in addition there are exaggerated sodium currents in the mutated channels [21, 22]. All these factors contribute to the pathogenesis of PE and PEPD, although it is not yet clear why certain regions in the body are more predisposed to pain development and why pain is paroxysmal [23]. It is also unclear why patients with PE respond well to cooling, which renders the threshold for pain comparable to people with wild-type Na1.7 channels.

CIP is a rare autosomal-recessive condition first described in patients from Pakistan presenting with insensitivity to all modalities of pain including visceral pain. CIP presents immediately after birth and is characterized mainly by insensitivity to pain which is the common feature among all affected individuals, although clinical heterogeneity exists, as some patients develop anosmia while others have intact smell sensation. Although the $\mathrm{Na} 1.7$ channels are expressed in the sympathetic nervous system, there is no autonomic nervous system instability, suggesting that $\mathrm{Na} 1.7$ channels have secondary roles at these sites or are compensated for by other channels.
The main differential diagnosis that should be considered is hereditary sensory neuropathy type 2 (HSAN2) (OMIM 201300), hereditary sensory neuropathy type 4 (HSAN4) (OMIM 256800) and hereditary sensory neuropathy type 5 (HSAN5) (OMIM 608654). Clinical features in these syndromes in addition to the loss of pain sensation include thermal and tactile abnormalities in addition to loss of neuropathic pain and autonomic nervous system instability [24]. Biopsies of the sural nerve in patients with these syndromes reveal loss of large and small myelinated fibers with some loss in unmyelinated fibers [25], in contrast to patients with CIP in whom the nerves are intact.

The SCN9A gene which encodes for Na1.7 channels is linked to chromosome 2q24. The protein is composed of 1,977 amino acids which form four similar domains. Each domain is composed of six transmembrane regions, with the channel pore being formed from the fifth and sixth transmembrane regions and the voltage sensor located in the fourth transmembrane segment of each domain. The domains are connected to each other by linkers that attach the sixth transmembrane segment of one domain to the first transmembrane segment of the next domain [7,25].

All mutations in the cases of CIP are homozygous nonsense $[7,17]$ mutations, except for one compound heterozygous mutation [16]. These mutations are expected to result in either nonsense-mediated mRNA decay with complete absence of channel formation, or to a truncated protein which lacks the normal protein function $[7,17]$.

Here, we identified a novel nonsense mutation in a Pakistani family, designated R523X, which is located in exon 10 and is part of the first linker which connects domain 1 to domain 2 . This site appears to be critical for the normal formation and function of the channel, since a previous mutation in the same linker designated S459X was associated with loss of function [7].

This mutation expands the body of evidence supporting the critical role of the Na1.7 channel in the perception of pain, and suggests that the absence of this channel cannot be compensated for by other channels.

In conclusion, we have extended the spectrum of mutations in Na1.7 channels in CIP, providing a framework for unraveling the complex mechanisms of pain perception.

\section{Acknowledgements}

We gratefully acknowledge the families for having participated in this study. We also thank Helen Lam for expert technical assistance. This study was supported in part by a research career development award from the Dermatology Foundation (Y.S.) and NIH grant from USPHS, NIH/NIAMS RO1 AR44924 (to A.M.C.). 


\section{References}

1 Waxman SG: The neuron as a dynamic electrogenic machine: modulation of sodiumchannel expression as a basis for functional plasticity in neurons. Philos. Trans R Soc Lond B Biol Sci 2000;355:199-213.

$\checkmark 2$ Isom LL: Sodium channel b subunits: anything but auxiliary. Neuroscientist 2001;7: 42-54.

3 Suls A, Claeys KG, Goossens D, et al: Microdeletions involving the SCN1A gene may be common in SCN1A-mutation-negative SMEI patients. Hum Mutat 2006;27:914920.

4 Sugiura Y, Makita N, Li L, et al: Cold induces shifts of voltage dependence in mutant SCN4A, causing hypokalemic periodic paralysis. Neurology 2003;61:914-918

5 Keller DI, Huang H, Zhao J, et al: A novel SCN5A mutation, F1344S, identified in a patient with Brugada syndrome and feverinduced ventricular fibrillation. Cardiovasc Res 2006;70:521-529.

-6 Trudeau MM, Dalton JC, Day JW, et al: Heterozygosity for a protein truncation mutation of sodium channel SCN8A in a patient with cerebellar atrophy, ataxia, and mental retardation. J Med Genet 2006;43:527-530.

$\checkmark 7$ Cox JJ, Reimann F, Nicholas AK, et al: An SCN9A channelopathy causes congenital inability to experience pain. Nature 2006;444: 894-898.

$\checkmark 8$ Drenth JP, te Morsche RH, Guillet G, et al: SCN9A mutations define primary erythermalgia as a neuropathic disorder of voltage gated sodium channels. J Invest Dermatol $2005 ; 124: 1333-1338$.
9 Fertleman CR, Baker MD, Parker KA, et al: SCN9A mutations in paroxysmal extreme pain disorder: allelic variants underlie distinct channel defects and phenotypes. Neuron 2006;52:767-774.

10 Nassar M A, Levato A, Stirling LC, et al: Neuropathic pain develops normally in mice lacking both Nav1.7 and Nav1.8. Mol Pain 2005;1:24.

11 Akopian AN, Souslova V, England S, et al: The tetrodotoxin-resistant sodium channel SNS has a specialized function in pain pathways. Nat Neurosci 1999;2:541-548.

12 Ahmad S, Dahllund L, Eriksson Ab, et al: A stop codon mutation in SCN9A causes lack of pain sensation. Hum Mol Genet 2007;16: 2114-2221

13 Dib-Hajj SD, Cummins TR, Black JA, et al: Nav1.7 and human pain disorders. Trends Neurosci 2007;30:555-563.

14 Yang Y, Wang Y, Li S, et al: Mutations in SCN9A, encoding a sodium channel alpha subunit, in patients with primary erythermalgia. J Med Genet 2004;41:171-174.

15 Drenth JP: The primary erythermalgia-susceptibility gene is located on chromosome 2q31-32. Am J Hum Genet 2001;68:12771282 .

16 Nilsen KB, Nicholas AK, Woods CG, et al: Two novel SCN9A mutations causing insensitivity to pain. Pain 2009;143:155-158.

17 Goldberg YP, MacFarlane J, MacDonald ML, et al: Loss-of-function mutations in the Nav1.7 gene underlie congenital indifference to pain in multiple human populations. Clin Genet 2007;71:311-319.

-18 Cummins TR, Howe JR, Waxman SG: Slow closed-state inactivation: a novel mechanism underlying ramp currents in cells expressing the hNE/PN1 sodium channel. J Neurosci 1998;18:9607-9619.
19 Renganathan M, Cummins TR, Waxman SG: Contribution of $\mathrm{Na}(\mathrm{v}) 1.8$ sodium channels to action potential electrogenesis in DRG neurons. J Neurophysiol 2001;86:629640 .

20 Sangameswaran L: A novel tetrodotoxinsensitive, voltage-gated sodium channel expressed in rat and human dorsal root ganglia. J Biol Chem 1997;272:14805-14809.

21 Harty TP, Dib-Hajj SD, Tyrrell L, et al: Nav1.7 mutant A863P in erythromelalgia: effects of altered activation and steady-state inactivation on excitability of nociceptive dorsal root ganglion neurons. J Neurosci 2005;26:12566-12575.

22 Han C: Sporadic onset of erythermalgia: a gain-of-function mutation in Nav1.7. Ann Neurol 2006;59:553-558

23 Drenth JP, Waxman SG: Mutations in sodium-channel gene SCN9A cause a spectrum of human genetic pain disorders. J Clin Invest 2007;117:3603-3609.

$>24$ Lafreniere RG, MacDonald ML, Dube MP, et al: Study of Canadian Genetic Isolates: Identification of a novel gene (HSN2) causing hereditary sensory and autonomic neuropathy type II through the Study of Canadian Genetic Isolates. Am J Hum Genet 2004;74: 1064-1073.

25 Hilz MJ: Assessment and evaluation of hereditary sensory and autonomic neuropathies with autonomic and neurophysiological examinations. Clin Auton Res Suppl 2002;12:33-43. 